\author{
Nota Científica \\ (Short communication)
}

\title{
HELIX ASPERSA (STYLOMMATOPHORA: HELICIDAE) PLAGA EMERGENTE DE LA MANZANA (MALUS SPP.) EN GUERRERO, CHIHUAHUA
}

\section{HELIX ASPERSA (STYLOMMATOPHORA: HELICIDAE) EMERGENT PEST OF THE APPLE (MALUS SPP.) IN GUERRERO, CHIHUAHUA}

\section{Isabel RUIZ-GalVÁN*, José Manuel PINEDA-RÍOS, Clemente de Jesús GaRCÍA-ÁVILA, DaniEL BRAVO-PÉREZ, JOSÉ GUSTAVO TORRES-MARTÍNEZ, JOSÉ GUADALUPE FLORENCIO-ANASTASIO, ANDRÉS QUEZADA-SALINAS, ISABEL VÁSQUEZ-LÓPEZ}

\begin{abstract}
Servicio Nacional de Sanidad, Inocuidad y Calidad Agroalimentaria. Unidad Integral de Servicios, Diagnóstico y Constatación, Tecámac, CP 55740, Estado de México, México <ruizg.isabel@gmail.com>; <pinedarmanuel@gmail.com>; <clemente.garcia@senasica.gob.mx>; <dabrape@live.com.mx>; <jose.torres@senasica.gob.mx>; <florenciojose@colpos.mx>; <andresqs@colpos.mx>; <isa628@gmail.com>

*Autor de correspondencia: <ruizg.isabel@gmail.com>
\end{abstract}

Recibido: 27/02/2018; aceptado: 17/05/2018; publicado en línea: 10/12/2018

Editor responsable: Jesús Romero Nápoles

Ruiz-Galván, I., Pineda-Ríos, J. M., García-Ávila, C. J., Bravo-Pérez, D., Torres-Martínez J. G., Florencio-Anastasio, J. G., Quezada-Salinas, A., Vásquez-López, I. (2018) Helix aspersa (Stylommatophora: Helicidae) plaga emergente de la manzana (Malus Spp.) en Guerrero, Chihuahua. Acta Zoológica Mexicana (nueva serie), 34, 1-5. https://doi.org/10.21829/azm.2018.3412161

RESUMEN. El manzano es uno de los principales frutales de importancia económica para México. Se ve afectado por diferentes plagas, una de ellas el caracol común de jardín (Helix aspersa), que se alimenta de hojas y fruta en desarrollo en la localidad de Mesa Miñaca, Guerrero, Chihuahua, desde 2013. A la fecha se desconocen las causas que han favorecido la presencia y dispersión de la plaga en esa localidad.

Ruiz-Galván, I., Pineda-Ríos, J. M., García-Ávila, C. J., Bravo-Pérez, D., Torres-Martínez J. G., Florencio-Anastasio, J. G., Quezada-Salinas, A., Vásquez-López, I. (2018) Helix aspersa (Stylommatophora: Helicidae) emergent pest of the apple (Malus Spp.) in Guerrero, Chihuahua. Acta Zoológica Mexicana (nueva serie), 34, 1-5. https://doi.org/10.21829/azm.2018.3412161

ABSTRACT. In Mexico the apple tree is one of the most important fruit-trees with high economical value. This tree is affected by several pests, one of them is the garden common snail (Helix aspersa). At the locality of Mesa Miñaca, Guerrero, Chihuahua, this snail feeds on apple leaves and also from its fruits. This behavior was observed since 2013 at this locality, and nowadays the causes of its emergence and dispersion remain unknown.

Los moluscos terrestres de la clase Gastropoda son de los más diversos del planeta, se calcula que existen 35,000 especies en el mundo (Solem, 1984) distribuidas en 112 familias (Barker, 2001). En México, se han 
reportado 47 familias y 1,184 especies y subespecies (Naranjo-García \& Fahy, 2010; Thompson 2011a, 2011b, 2012), lo que representa el 3.4\% de la diversidad mundial. En México, se ha dado mayor atención a estudios de investigación de los moluscos marinos, omitiendo a los terrestres y dulceacuícolas, a pesar de la importancia que tienen en la economía y la salud (Naranjo-García, 1994). Helix aspersa (Müller, 1774) (Stylommatophora: Helicidae) (Wade et al., 2001) se reproduce con fines comerciales (Lamprea et al., 2009; Díaz et al., 2007), pero puede convertirse en plaga agrícola o ser transmisor y vector de enfermedades en artrópodos y plantas (Cruz et al., 2002; Álvarez et al., 2009). En algunos casos, se comporta como especie invasora por las altas poblaciones y daños que ocasiona en la citricultura, viticultura, horticultura y jardinería (Jørgensen \& Sørensen, 2008). En cítricos de California, EE.UU., llega a causar del 40 al 50 o incluso hasta el 100\% de pérdidas en producción, aumentando su población en años lluviosos, especialmente cuando éste ocurre en los meses calurosos (Sakovich, 2002); en regiones de Australia y Sudáfrica, se aloja en los tallos, follaje y racimos de vid (Sanderson \& Sirgel, 2002).

En 2013, en tres hectáreas de plantación de manzano en la localidad de la Mesa Miñaca, Guerrero, Chihuahua, México, se observaron pequeñas poblaciones de caracoles sin evidencia de daños en las plantas; tres años después, la población de estos caracoles aumentó, se registraron daños y se dispersó aproximadamente a 50 hectáreas (Hernández-Enríquez E., comunicación personal, 9 de junio de 2016).

Derivado de este problema fitosanitario, el Comité Estatal de Sanidad Vegetal de Chihuahua, notificó al Servicio Nacional de Sanidad, Inocuidad y Calidad Agroalimentaria (SENASICA) a través de la Dirección General de Sanidad Vegetal (DGSV) la necesidad de determinar la especie de molusco y establecer acciones de control. En junio de 2016, se realizó una recolecta de caracoles en el huerto Tío Simón, ubicado en las coordenadas 28.516094, -107.457420 a una altura de $2090 \mathrm{msnm}$. Las muestras incluyeron caracoles de cualquier etapa de desarrollo, tomados al azar y localizados en el dosel de los árboles de manzano y en el suelo. Los especímenes se recolectaron manualmente con guantes de látex, se colocaron vivos en frascos de plástico con perforaciones y hojas frescas de manzano para su alimentación, con la finalidad de mantenerlos vivos para poder llevar a cabo el diagnóstico. Se transportaron al Centro Nacional de Referencia Fitosanitaria (DGSV-SENASICA) y posteriormente al Departamento de Zoología del Instituto de Biología, de la Universidad Nacional Autónoma de México (Ciudad de México), con la Dra. Edna Naranjo García, especialista en moluscos terrestres, para su determinación. La especie fue identificada como Helix aspersa conocida comúnmente como "caracol de jardín".

Derivado de observaciones realizadas y de la información obtenida en 2016 y 2017, en la localidad de Mesa Miñaca, se determinó que los caracoles juveniles y adultos comienzan a emerger a inicios de marzo y las poblaciones se redujeron en noviembre. En junio y agosto se observaron masas con 76 huevos en promedio de 30 observaciones, depositadas éstas alrededor del tronco de los árboles de manzano, entre 1 a $2 \mathrm{~cm}$ de profundidad del suelo; en ocasiones también se les observó refugia en malezas presentes en las huertas de manzano. En 2016, se observaron en altas infestaciones en los meses de junio a agosto, se cuantificaron hasta 1,000 caracoles/árbol. Inicialmente, $H$. aspersa, se alojó en el tronco del árbol y bajo las ramas (Fig. 1), posteriormente se desplazó a la copa para alimentarse del follaje y realizar perforaciones irregulares en los bordes y centro de las hojas, en los frutos hizo perforaciones irregulares, generalmente en la parte basal de los mismos. Este daño es parecido al que causan algunas larvas de insectos; sin embargo, se diferencia por el rastro que va dejando del mucus. No se observaron preferencia de alimentación por alguna variedad de manzano.

A pesar de ser una plaga polífaga, no se han documentado casos de daño en manzano u otro cultivo de importancia económica en México, por lo que este es el primer reporte de daños causados por H. aspersa en el cultivo de manzano en el país.

Ante esta problemática, las acciones fitosanitarias de manejo y control se han enfocado en la recolección manual y destrucción de huevos, juveniles y adultos; además de eliminación de sitios de refugio 
(madrigueras de roedores, madera, rocas, restos de cosecha, leña, etc.), aplicación de caldo bordelés en el tallo y el uso de trampas metálicas colocadas en el tallo (Fig. 2), estas últimas han impedido la colonización de caracoles en la parte aérea del árbol y evitan pérdidas considerables de producción.

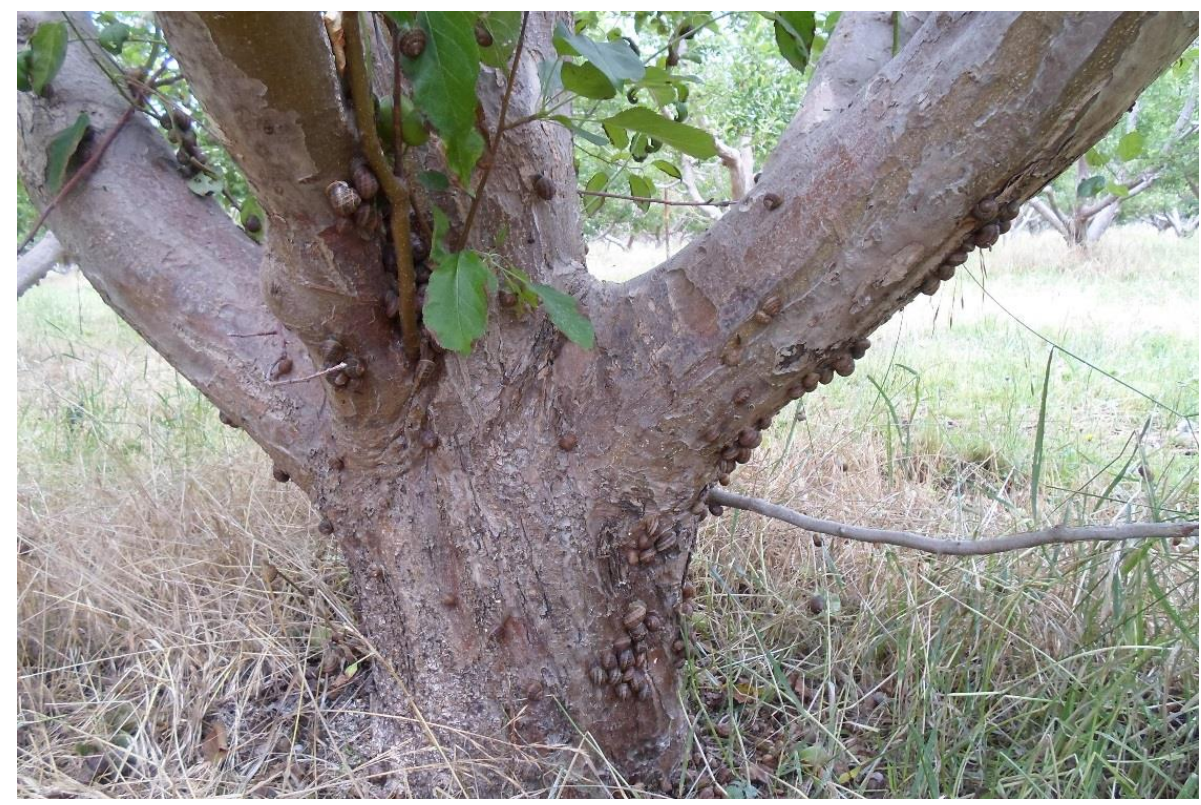

Figura 1. Alta infestación de Helix aspersa alojados en troncos y ramas de un árbol de manzano.

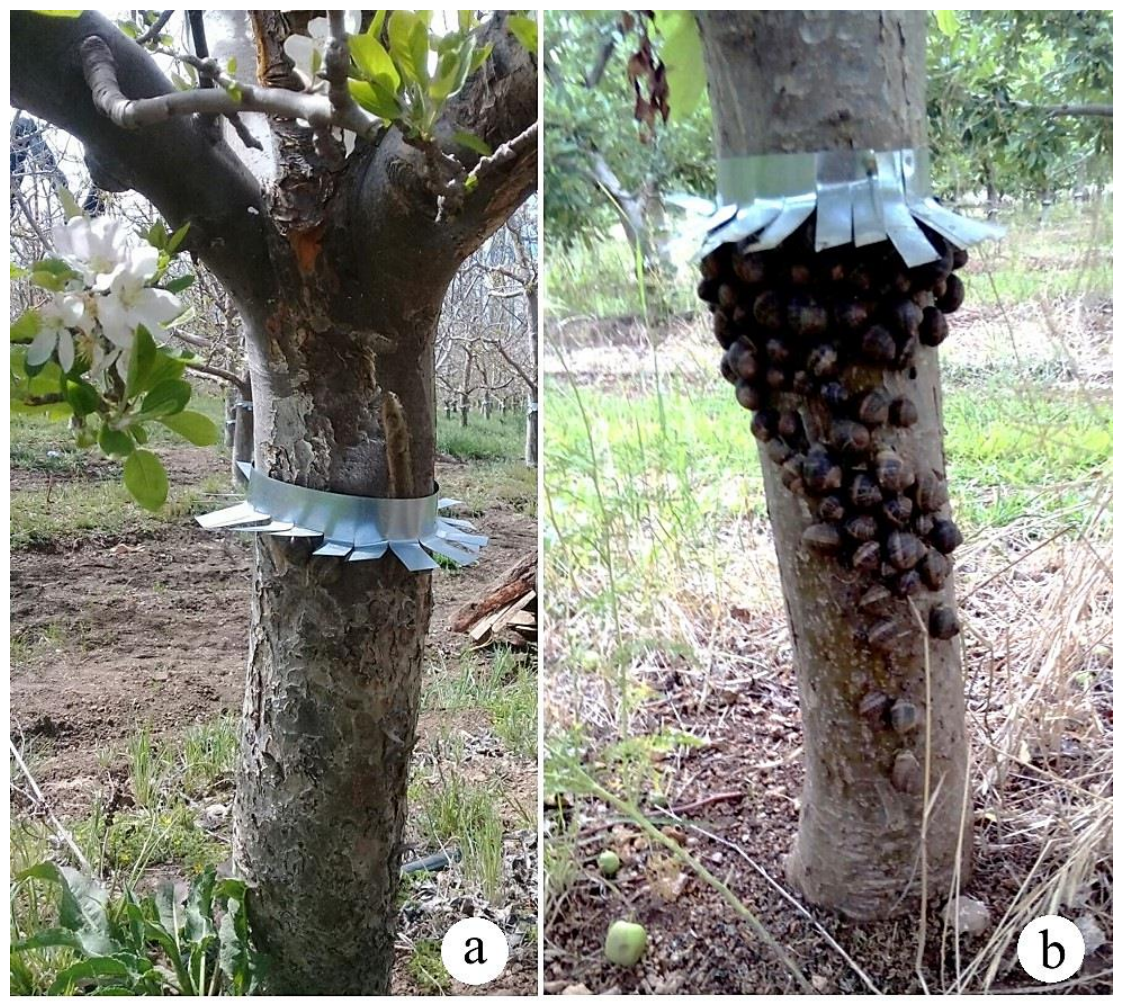

Figura 2. a) Barrera de lámina para el control de caracoles; b) Caracoles retenidos en la barrera física. 
Letelier (2005) citó que gran parte de los caracoles terrestres que causan grandes daños económicos en la agricultura tienen en común el haber llegado accidentalmente a un lugar. En México, H. aspersa no se considera una plaga de importancia económica en la agricultura; se desconoce la causa que originó su presencia y cómo se ha incrementado su población; aunque Ramírez et al. (2011) mencionaron que en zonas manzaneras de Chihuahua, la presencia y aumento de nuevas plagas se debe a las variaciones climáticas. A la fecha no existen evidencias específicas para sostener esta teoría. Por lo anterior, existe la necesidad de realizar un estudio biogeográfico para determinar su distribución y fluctuación poblacional en algunas regiones del país, así como los factores que promueven su dispersión y establecimiento en nuevas áreas, aspectos de ecología, dinámica poblacional y acciones de manejo y control.

Agradecimientos. Se agradece al Comité Estatal de Sanidad Vegetal de Chihuahua y al Ing. Leonel Nájera Frescas, por el apoyo en los recorridos de campo y por la información proporcionada.

\section{LITERATURA CITADA}

Álvarez, L. A., Gramaje, D., Abad, C. P., García J. J. (2009) Role of the Helix aspersa snail as a vector of Phytophthora citrophthora causing branch cankers on clementine trees in Spain. Plant Pathology, 58, 956-963.

Barker, G. M. (2001) Gastropods on land: phylogeny, diversity and adaptive morphology. Pp.1-46. In: G. M. Barker (Ed.). Chapter 1. The Biology of Terrestrial Molluscs. CAB International, Wallingford, UK.

Cruz, M. I., Ibarra, V. F., Naranjo, G. E., Quintero, M. M. T., Lecumberri, L. J. (2002) Identificación Taxonómica, estacionalidad y grado de infección con Fasciola hepatica de moluscos huéspedes y no huéspedes intermediarios del trematodo en el rancho de la Universidad Autónoma de Hidalgo. Universidad Autónoma de Hidalgo, México, 12 pp.

Díaz, J. I., Aguirre, J. C., Mejía, G., Martínez, G. E. (2007) Reproducción y genética del caracol terrestre "Helix aspersa". Revista CES / Medicina Veterinaria y Zootecnia, 2, 78-88.

Jørgensen, S. P., Sørensen, N. (2008) The Invasive Potential of the Brown Garden Snail (Cantareus aspersus): A Future Invasive Species in Denmark? University of Copenhagen. Denmark, 84 pp.

Lamprea, D. A., Salazar, A. M. C., Posada, A. A. (2009) Formulación de la cadena productiva del caracol en Cundinamarca y Boyacá-Colombia para exportar hacia el mercado español. Revista U.D.C.A Actualidad \& Divulgación Científica, 12, 163-172.

Letelier, S. V. (2005) Antecedentes sobre especies introducidas en Chile: Monitoreo y Control. Boletín AMICI 2005. Sociedad Malacológica de Chile, 14-16.

Naranjo-García, E. (1994) Estudios de los moluscos terrestres en México. In: Naranjo-García E., M. T. Olivera y O. J. Polaco (Comps.). Seminario: La situación actual de la Malacología Médica y Aplicada en Latinoamérica. Sociedad Internacional de Malacología Médica y Aplicada. México.

Naranjo-García, E., Fahy, N. E. (2010) The Lesser Families of Mexican Terrestrial Molluscs. American Malacological Bulletin, 28, 59-80.

Ramírez, L. M .R., Ruiz, C. J. A., Medina, G. G., Jacobo, C. J. L., Parra, Q. R. A., Avila, M. M. R., Pila, J. (2011) Perspectivas del sistema de producción de manzano en Chihuahua, ante el cambio climático. Revista Mexicana de Ciencias Agrícolas, 2, 265-279.

Sakovich, N. J. (2002) Integrated Management of Cantareus aspersus (Müller) (Helicidae) as a Pest of Citrus in California. Pp.353-360. In: G. M. Barker (Eds.). Molluscs as crop pests. CAB International, London, UK.

Sanderson, G., Sirgel, W. (2002) Helicidae as Pests in Australian and South African Grapevines. Pp. 255-270. In: G.M. Barker (Eds.). Molluscs as crop pests CAB International, London, UK.

Solem, A. (1984) A World model of land snail diversity and abundance. Pp. 6-22. In: A. Solem y A. C. van Bruggen (Eds.), World-wide snails. Biogeographical studies on non-marine Mollusca E. J. Brill, Leiden, The Netherlands. 
Thompson, F. G. (2011a) Mexistrophia, a new genus of Cerionidae from Mexico (Gastropoda: Pulmonata: Urocoptoidea). The Nautilus, 125, 182-192.

Thompson, F. G. (2011b) An annotated checklist and bibliography of the land and freshwater snails of Mexico and Central America. Bulletin of the Florida Museum of Natural History, 50, 1-299.

Thompson, F. G. (2012) The land snail genus Epirobia and allied genera in Mexico and Central America, with description of a new family, the Epirobiidae (Gastropoda, Pulmonata, Urocoptoidea). Bulletin of the Florida Museum of Natural History, 51, 167-215.

Wade, C. M., Mordan, P. B., Clarke, B. (2001) A phylogeny of the land snails (Gastropoda: Pulmonata). Proceedings of the Royal Society B: Biological Sciences, 268, 413-422. 